\title{
VIVIR EN SITUACIÓN DE CALLE EN CONTEXTOS URBANOS: SUBJETIVIDADES EN RESISTENCIA
}

\author{
Jorgelina Di Iorio ${ }^{l}$ \\ Consejo Nacional de Investigaciones Científicas y Tecnológicas, Argentina \\ Universidad de Buenos Aires, Argentina
}

\begin{abstract}
RESUMEN
Vivir en situación de calle constituye uno de los modos en que se institucionalizan los procesos de vulnerabilización social en los contextos urbanos. La invisibilización de la problemática se expresa en las tensiones en lo que respecta a la delimitación del problema y a las metodologías utilizadas para relevarlas. En este artículo se describe un proceso comunitario de relevamiento territorial de amplio alcance realizado en la Ciudad de Buenos Aires (Argentina), a partir de la utilización de metodologías participativas. Se problematizan las dimensiones instrumentales y las ético-políticas del relevamiento, entendido como intervención psicosocial, en clave de co-construir territorios de intervención que promuevan transformaciones subjetivas y colectivas, que se traduzcan en la ampliación de derechos.
\end{abstract}

\section{Palabras claves}

personas en situación de calle -metodologías participativas- dimensión ético-político

\section{Abstract}

Homelessness is one of the ways in which social vulnerability processes are institutionalized in urban contexts. The invisibility of the problem is expressed in the tensions regarding the delimitation of the problem and the methodologies used to survey them. This article describes a community process of wideranging territorial survey carried out in the City of Buenos Aires (Argentina), based on the use of participatory methodologies. The instrumental and ethical-political dimensions of the survey are problematized, in order to co-construct territories of intervention that promote subjective and collective transformations, which translate into the extension of rights.

\section{Keywords}

homeless people - participatory methodologies - ethical-political dimension

\footnotetext{
1 Correspondece about this article should be addressed to Jorgelina Di Iorio. Co-directora de Proyecto UBACyT 2018-2020 Sociogénesis de las marginaciones urbanas: personas en situación de calle en la Ciudad de Buenos Aires financiado por la Universidad de Buenos Aires. E-mail: diiorio.jorgelina@gmail.com
} 


\section{LIVING IN A STREET SITUATION IN URBAN CONTEXTS: SUBJECTIVITIES IN RESISTANCE}

Resulta difícil negar la sensibilidad e incluso preocupación que generan las personas en situación de calle en los contextos urbanos. En particular, con la llegada de las bajas temperaturas, las personas en situación de calle comienzan a ser vistas, aunque no reconocidas: notas periodísticas e informes que, con descripciones simples, a la vez que conmueven a la audiencia reducen la complejidad del campo de problemas: la indignación facebuquera que denuncia en 280 caracteres o que los convierte en trending topic hasta que aparezca una nueva noticia; diseño e implementación de programas gubernamentales focalizados; estudios o investigaciones con pretensión de explicación del fenómeno. La angustia, el dolor, la indignación, el miedo aparecen como disposiciones emocionales que orientan la reflexión sobre estos otros -quienes están en situación de calle- como víctimas de formas arbitrarias de violencias.

Estar en situación de calle, personas de la calle, habitantes de calle, deambulantes, personas sin hogar, homeless, son algunos de los términos que se encuentran en la literatura internacional para hacer referencia a una problemática social compleja. No se reduce a quienes literalmente utilizan el espacio público como lugar de pernocte, sino que se refiere a un fenómeno que está atravesado por dimensiones culturales, políticas, históricas, sociales y económicas. "Estar en situación de calle" se define como una paradójica forma de inclusión social sostenida desde la marginalización, la ruptura y/o fragilidad de vínculos sociales, laborales y familiares, por las dificultades para cubrir necesidades materiales, simbólicas y afectivas, así como también por la vulneración de derechos sociales, económicos y culturales. La calle constituye un espacio de vivencia, supervivencia y resistencia en un continuo proceso de posesióndesposesión material, simbólico y afectivo. Además de déficit de vivienda y de trabajo, acumulan otro conjunto de vulnerabilidades psicosociales entre las que se incluyen debilitamiento de la red socio-familiar de apoyo, aislamiento social, padecimientos físicos y de salud mental, exposición a violencias, así como también dificultades en el acceso a derechos sociales, culturales y políticos (Boy, 2012; Pallares, 2012; Seidmann et al 2009) Pese a lo que pudiera considerarse desde una mirada ingenua, la vida cotidiana de quienes están en situación de calle está organizada a partir del despliegue de un conjunto de secuencias preestablecidas, que delimitan hábitos y rutinas en el uso del espacio público. Siguiendo a Di Iorio et. al. (2014, p.5, "sus trayectorias configuran nuevas territorialidades de lo público, expresando subjetividades que no pueden ser reducidas a la objetividad del mercado", en las que esos cuerpos desechables y condenables por peligrosos, resignifican el uso del espacio público urbano estableciendo otros sentidos a un escenario social que les obtura la entrada.

Relevar las poblaciones en situación de calle es parte de las acciones de gobiernos y de organizaciones de la sociedad civil en los grandes centros urbanos en todo el mundo. Los modos en que esos relevamientos tradicionalmente son realizados revelan limitaciones en lo que respecta a la delimitación del área a relevar, los costos y el financiamiento, la definición de situación de calle como objeto-sujeto de estudio y el uso posterior de los datos (Cowan, 1991). En la Ciudad Autónoma de Buenos Aires (Argentina), desde el año 1997 se realiza un conteo durante una noche al año para dar cuenta de la problemática. Sin embargo, organizaciones sociales y de derechos humanos (Donda, 2016) vienen denunciando la necesidad de implementar metodologías acordes a la problemática, que permitan dar cuenta de su dinámica y su complejidad, tal como establece la Ley $N^{\circ} 3706 / 11$ de Protección de derechos de las personas en situación de calle (2011) En este sentido, se presenta en este articulo el caso del 1er Censo Popular de Personas en Situación de Calle realizado en la Ciudad Autónoma de Buenos Aires en el año 2017, con la intención de presentar una metodología alternativa organizada a partir de los conceptos de participación, territorio y poder popular. No se pretende dar cuenta de los resultados, ya que los mismos fueron publicados por las organizaciones que lo coordinaron, sino en tipo de relevamiento que puede ser comprendido como una intervención comunitaria.

\section{Delimitación del campo de intervención}

a. (Re)Definir el problema, (Re)Pensar las respuestas

El escenario social globalizado y de economías concentradas, transnacionalizadas y desnacionalizadas que caracteriza a América Latina y al mundo configura formas específicas de 
marginalidad urbana que se mantienen en ascenso desde la década del '70 (Wacquant, 2010; Kessler \& Merklen, 2013). Se moderniza miseria: ascenso de la desigualdad sectorizada espacialmente en los contextos urbanos. Se hacen visibles grupos sociales constituidos en los padecimientos de su pertenencia a un todo social fragmentado, que se traduce en una forma de opresión y dominación caracterizada por la vulneración de derechos. Se trata de un fenómeno crónico del contexto urbano que genera desigualdades durables como un continum exclusión-inclusión (Bustelo \& Minujin, 1997) o de inclusión desde la marginalidad (Sawaia, 2011). Estos grupos sociales, definidos como underclass urbana o parias urbanos (Wacquant, 2010), se configuran en función de complejas problemáticas sociales, entre las que se encuentra la situación de calle.

Estar en situación de calle no es un estado o una cosa, sino una relación social, donde lo efímero se convierte en constante, emergiendo una forma de padecimiento social relacionada con expresiones de inequidad e injusticia social, configurándose identidades estigmatizadas (Di Iorio et. al. 2017). Pese a ser un grupo social heterogéneo, es homogeneizado al diseñar programas de intervención en función de su condición de privación y exclusión producto de un proceso continuo de posesión y desposesión material, simbólica y afectiva. Esto los hace poseedores de atributos socialmente desacreditadores, dando lugar a procesos de estigmatización (Goffman, 2003). La profundización de la distancia social entre estos grupos sociales y el resto de la sociedad da lugar a que sean vistos como socialmente amenazantes, culturalmente estigmatizados y económicamente marginales. Es decir, la situación de calle como problema social complejo constituye una de las formas institucionales en las que se expresan los procesos de vulnerabilización y expulsión en los contextos urbanos, entendida en términos de complejas relaciones entre diferencias económicas, desigualdades jurídicas y desafiliaciones sociales (Di Iorio, 2016).

La problemática no se reduce a quienes literalmente utilizan el espacio público como lugar de pernocte (situación de calle efectiva), sino que incluye todo otro conjunto de personas que utilizan la red de alojamientos nocturnos transitorios -hogares y paradores, ya que no constituyen una respuesta definitiva a la situación habitacional de las personas ${ }^{2}$. Siguiendo a Crenshaw (1995 citado en Molina, 2013) no se trata de una suma de desigualdades, sino que cada una de éstas intersecciona, se cruza, de forma diferente en cada grupo social mostrando estructuras de poder existentes en el seno de la sociedad. Es decir, impacta en las configuraciones subjetivas, pero también en el modo en que se definen y abordan estas desigualdades.

b. La importancia de los relevamientos: Visibilizar para humanizar

No saber cuántas personas en situación de calle, así como subregistrarlas constituye una de las formas en las que se expresa la violencia del Estado. Y tan importante como las cifras son los datos cualitativos que permiten relevar las necesidades concretas de esta población, cómo estas personas se autoperciben, su estado de salud, su trayectoria educativa y los tipos y niveles de violencia institucional que padecen, entre otras cosas.

Desde 1997, el gobierno local de la Ciudad de Buenos Aires realiza un conteo sobre PSC. Tal como se observa en el gráfico, el dato se mantiene sin muchas variaciones hasta la actualidad. En 2016 el Gobierno de la Ciudad (GCBA) registró 876 personas en esa situación, casi lo mismo que en el 2012 y 2014, y apenas unas 100 personas menos que lo que registró en el 2015 (n=924). En 2017 el gobierno local reconoció un aumento respecto del año anterior, contabilizando 1066 personas durmiendo en el espacio público (La Nación, 2017)

\footnotetext{
2En la Ciudad de Buenos Aires también se incluyen a quienes están en riesgo de situación de calle. Según la Ley N³706/11 están en riesgo de calle: 1. personas con sentencia de desalojo, 2. personas que viven en hoteles bajo el subsidio habitacional otorgado por el Decreto 690/GCABA/06 y sus modificatorios o amparos, 3 . quienes duermen en estructuras precarias temporales o asentamientos, 4. personas institucionalizadas en cárceles, hospitales generales y hospitales monovalentes (salud mental) con posibilidad de egreso, jóvenes por cumplir 18 años institucionalizados en el sistema de protección con pronto egreso. A esto se suman las diferencias por género, por edad y por etnia.

- Al momento de escribir este artículo se está llevando a cabo el 2 do Censo Popular, encontrándose en la etapa de procesamiento de datos. Asimismo, en la misma fecha se realizó el conteo gubernamental/oficial que dio 1146 personas. http://elgritodelsur.com.ar/2019/06/segun-el-gobierno-portenohay-solo-55-personas-mas-en-la-calle-que-hace-un-ano.html
} 


\section{Gráfico 1}

Total de PSC relevadas anualmente por GCBA

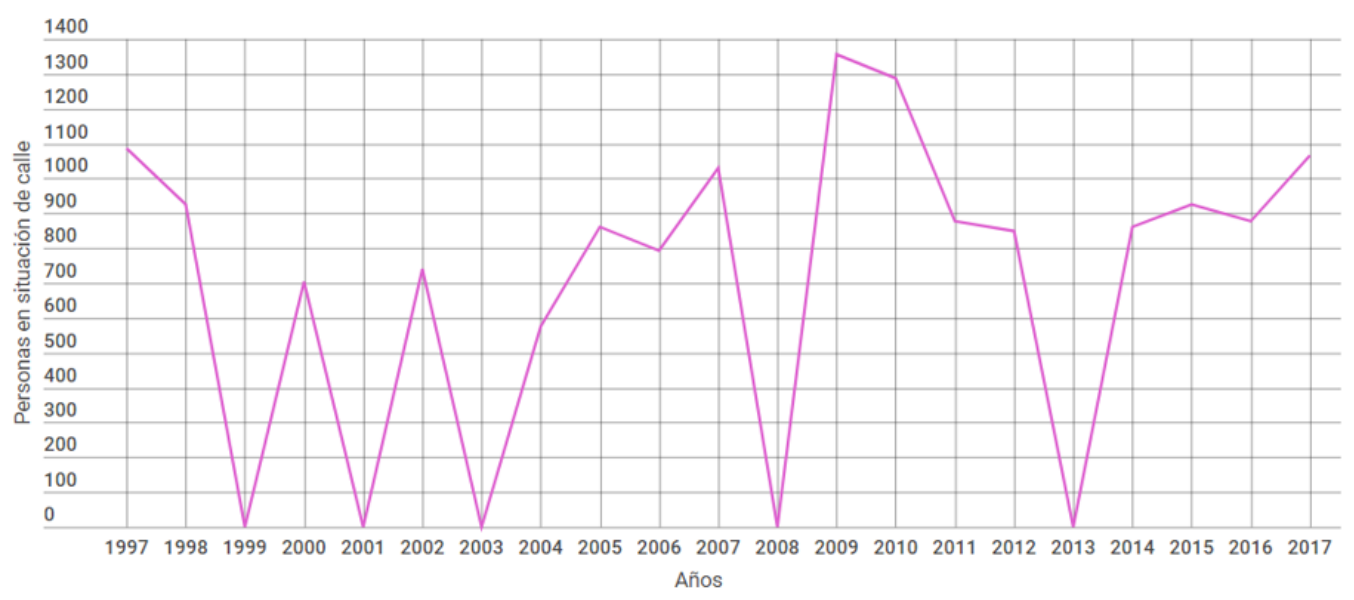

Fuente de elaboración propia por estudiantes de la Carrera de Comunicación Social a partir de datos publicados por el GCBA. Cuando el valor indica cero alude a la falta de dato.

Esta invariabilidad es denunciada por organizaciones sociales y políticas así por organismos de derechos humanos de escala local desde hace años. No condice con el aumento de la tasa de pobreza $(32,2 \%)$ y de indigencia $(6,3 \%)$ registrado para el área metropolitana en el último año (Pontificia Universidad Católica Argentina, 2017) ni tampoco con el deterioro socio-económico admitido por las propias cifras oficiales que registraron para el período enero 2016-mayo 2017 un aumento de la pobreza al $18,9 \%$ y de la indigencia al 5\% (Censos GCBA, 2017), como producto de un modelo económico definido por el aumento de la inflación, devaluación de la moneda, despedidos masivos, aumento de impuestos, vaciamiento del Estado y endeudamiento con organismos internacionales. El estancamiento de la actividad económica y la restricción y vulneración de derechos generaron un aumento de la pobreza que se registra en las calles, tal como sostienen quienes asisten a esta población.

Sobre el tipo de diseño utilizado oficialmente, Rosa (2012) explica que consiste en la localización, conteo y apreciación visual del sexo y edad aproximada, por observación y sin tomar contacto con las personas. No contempla la variabilidad de recorrido y de localización de las personas en situación de calle, producto de las propias condiciones de vida. En el 2016, y frente a la presión ejercida por organizaciones sociales, se realiza una denuncia en el Juzgado en lo contencioso y administrativo $\mathrm{N}^{\circ} 4$ para exigir al Gobierno de la Ciudad realizar un relevamiento que incluya en su diseño y ejecución a organizaciones de la sociedad civil, a las universidades y al gobierno (Donda, 2016). Asimismo, desde el Consejo Económico y Social, un organismo descentralizado de la Ciudad de Buenos Aires, se hicieron recomendaciones respecto de la aplicación de la ley, que no fueron escuchadas. Y si bien, la respuesta de la justicia (2017) fue favorable para las organizaciones en tanto que se cuestionan las cifras oficiales y se obliga al gobierno local como órgano de aplicación de la Ley $\mathrm{N}^{\circ} 3706$, a realizar un nuevo relevamiento con otra metodología e incorporando a las organizaciones que trabajan en el tema, se continúa realizando por observación, sin tomar contacto con las personas, sin relevar datos cualitativos y sin incluir al conjunto de organizaciones que trabajan en la temática.

La persistencia en la invisibilización, el aumento de la criminalización y represión, el aumento exponencial de PSC y la no convocatoria a una mesa de diálogo, dio origen a la conformación de una mesa de trabajo para la realización de un relevamiento diseñado e implementado de manera conjunta entre organizaciones de la sociedad civil que vienen abordando la problemática, expertos en la materia y personas en situación de calle o en riesgo a la situación de calle, tal como establece la Ley. Esto no significa que el Estado ignora la problemática, sino que la invisibiliza, lo que constituye una forma de violencia institucional, ya que: 
- No incluye en su conteo de personas en situación de calle a quienes se alojan en forma transitoria en la red de alojamiento nocturno, desconociendo el artículo 2 inciso "a" de la Ley $\mathrm{N}^{\circ}$ 3706/11

- No recorre la totalidad de los barrios en que está organizada la Ciudad, sino que registra en "zonas críticas"

- No contempla que la población en situación de calle es dinámica e itinerante en el diseño metodológico que propone.

- No incluye a las organizaciones sociales, políticas y comunitarias que trabajan con el tema

- No publica los datos que tiene

\section{Método}

\section{Un procedimiento centrado en la participación, el territorio y el poder popular}

Partiendo de la distancia entre los datos oficiales y el registro de las organizaciones que trabajan en la temática, se realizaron durante los meses de marzo y abril de 2017 reuniones semanales en las que surgieron los siguientes interrogantes: ¿Cómo se explica esa diferencia en el número? ¿No fue contabilizada o no existe? ¿Por qué no fue relevada? ¿Cuántas personas en situación de calle hay en la Ciudad de Buenos Aires?

Siguiendo a Cowan (1991), los relevamientos para dar cuenta de la cantidad de PSC implican ciertos desafíos metodológicos que se traducen en resultados e impactos diferentes: 1. Definición de la población a censar; 2. la itinerancia de la población como constitutiva de la organización de su vida cotidiana; 3. el financiamiento. El modo en que se resuelvan no se limita a aspectos técnicos, sino a aspectos ético-políticos. La dimensión ético-política (Goltzman \& Di Iorio, 2012) en tanto que ética de la relación y política para la transformación, supone problematizar las relaciones de subaternidad con los grupos en condición de vulnerabilidad y los modos de incluirlos en la producción de conocimientos, al para qué de esos conocimientos y a la potencialidad de los mismos para la reducción de la inequidad e injusticia social. En relación con la definición, el relevamiento oficial, considera población a ser contada aquella que se encuentre a la intemperie al momento del relevamiento. No incluye a quienes se encuentran en la red de alojamiento nocturno transitorio, que incluye refugios gubernamentales y hogares del tercer sector conveniados. En el caso del Censo Popular (2017), se considera situación de calle efectiva a quienes están en el espacio público y quienes están en los dispositivos de alojamiento, tal como establece la Ley $\mathrm{N}^{\circ}$ $3706 / 11$

La itinerancia de la población alude al movimiento que implica la subsistencia. Los usos y sentidos de la ciudad se organizan para las PSC según días y horarios, según el momento del año (Di Iorio et al 2014). Es decir, se configuran circuitos y trayectorias que requieren metodologías de achique o que promuevan el encuentro. Además, las personas en situación de calle constituyen lo que algunos autores denominan poblaciones ocultas o de difícil acceso (Lambert \& Wibel, 1990). Una población es considerada de difícil acceso si tuviera por lo menos uno de los siguientes atributos: ser rara (poco frecuente, geográficamente concentrada o dispersa), ser oculta (por comportamientos ilegales o especiales) $\mathrm{o}$ fluctuante (intermitencia de su asociación a puntos concretos del espacio geográfico) (Bastos \& Bertoni, 2014) Esto significa que frente al rastreo por zonas críticas (algunos lugares fijos en la Ciudad) que se realiza de modo oficial, el Censo Popular (2017) fue relevamiento exploratorio de amplia cobertura y alcance, basado en un tipo de muestreo coincidental. El muestreo de tipo coincidental o accidental, es una técnica de muestreo no probabilística basada en la accesibilidad y la disponibilidad de las unidades de análisis que conforman la población en estudio (Ochoa, 2015). Además, el relevamiento duró una semana y se debía garantizar pasar por los mismos lugares en distintos momentos. En relación con este aspecto y la dimensión territorial se aborda más adelante.

De acuerdo con esto, fueron contactados aquellos hombres, mujeres o personas trans mayores de 18 años y grupos familiares que se encontraran en situación de calle al momento en que fueron recorridos 
los 48 barrios en los que se organiza la Ciudad de Buenos Aires. Las personas participaron de manera voluntaria, pudiendo interrumpir o dejar de contestar en cualquier momento el cuestionario.

Finalmente, este tipo de relevamiento de amplio alcance, supone un costo alto en términos de financiamiento, que por lo general no están disponibles desde organismos públicos-estatales: recursos materiales, recursos humanos para hacer el trabajo de campo, recursos humanos para el procesamiento de los datos, infraestructura para el procesamiento. El Censo Popular se garantizó desde un conjunto de organizaciones sociales, comunitarias y políticas legitimadas por la población en situación de calle ya que trabajan con ella desde hace mucho tiempo. En este sentido, los recursos humanos y materiales fueron parte de su tarea social en términos de construcción colectiva de poder popular. Cada organización en la medida de sus posibilidades colaboró con los insumos necesarios, así como localizaciones físicas para poder hacer de base operativa durante la realización del relevamiento. Tanto la Defensoría del Pueblo como el Ministerio Público de la Defensa, en tanto organismos de gobierno de exigibilidad de derechos y que funcionan como contralor de la gestión pública y que fueron parte del Censo Popular, realizaron la carga de datos y el primer procesamiento de los mismos con el programa estadístico SPSS, por contar con esos recursos y con personal técnico disponible.

El proceso de diseño participativo se organizó en distintas etapas: 1. Conformación mesa de organizaciones participantes y convocatoria, 2. Construcción del instrumento, 3. Delimitación del recorrido, 4. Entrenamiento de "censistas", y 5. Construcción del informe.

\section{Convocatoria y Participantes}

Durante los meses de febrero y marzo de 2017, un conjunto amplio de organizaciones, entre las que se incluyen personas en situación de calle, que desde hace varios años son parte de la red socio-asistencial para personas en situación de calle de la Ciudad de Buenos Aires: Abrigar Derechos; Acción Voluntaria; Amigos en el Camino; Asamblea Popular Plaza Dorrego; Centro de Estudiantes de la Facultad de Psicología de la UBA; Centro de Integración Frida; Centro de Integración Monteagudo; Che Cultura; Ciudad Sin Techo; Comedor El Gomero de Barrancas de Belgrano; Comunidad del Centro Educativo Isauro Arancibia; Consejo de Organizaciones Sociales de la Defensoría del Pueblo de la Ciudad de Buenos Aires; Equipo de Investigación UBACyT 20020130100089BA de la Facultad de Psicología (UBA); Izquierda Popular; Juventud Socialista Democracia y Participación; La Boca Resiste y Propone; La Calle Que Nos Parió; La Miguelito Pepe; Movimiento Barrios de Pie; Movimiento Evita; Movimiento Libres del Sur; Movimiento Universitario Sur; Mujeres Activando; Mujeres de la Matria Latinoamericana (Mumalá); Ni una persona más en la calle; No Tan Distintas - Mujeres en Situación de Vulnerabilidad Social; Nuevo Encuentro; Observatorio del Derecho a la Ciudad; Partido Social; Patria Grande; Presidencias de las comisiones "Derechos Humanos, Antidiscriminación y Garantías" y "Mujer, Infancia, Adolescencia y Juventud" de la Legislatura de la CABA; Proyecto 7 - Gente en situación de calle; Red Puentes (MP La Dignidad); Sopa de Letras; Un Paso Hacia El Cambio (UPHEC). Asimismo, fueron parte del relevamiento el Ministerio Público de la Defensa de la Ciudad de Buenos Aires, la Defensoría del Pueblo y la Presidencia de la Auditoría General de la Ciudad de Buenos Aires. Estos organismos gubernamentales se caracterizan por no ser órgano de aplicación de política pública sino por su condición de exigibilidad de derechos. Es decir, constituyen la red de organismos públicos que debe exigir al Gobierno de la Ciudad el pleno ejercicio de los derechos, funcionando como auditores de política pública.

\section{Construcción del instrumento}

Se construyó para la recolección de datos una encuesta o cuestionario con preguntas cerradas, diseñada para aplicar a partir de la realización de una entrevista breve o conversación con las personas en situación de calle. El formulario se diseñó para relevar la situación de calle efectiva en el espacio público: hombres o mujeres adultos/as o grupo familiar, sin distinción de género u origen que habiten en la calle o espacios públicos de la Ciudad Autónoma de Buenos Aires en forma transitoria o permanente y/o que utilicen o no la red de alojamiento nocturno (Ley $\mathrm{N}^{\circ} 3706 / 11$ Art. 2). Las PSC que estuvieran en la red de alojamiento durante el Censo Popular, fueron relevadas con datos secundarios a partir de pedidos de información según la Ley $\mathrm{N}^{\circ} 104$ - Solicitud de información pública. 
El formulario tiene una parte observacional a ser completada por quien censaba y otra con un cuestionario cuyas respuestas fueron voluntarias y que permitió obtener información cualitativa. El registro observacional incluyó la ubicación geográfica y otros datos sobre la persona contactada: características demográficas básicas (sexo y estimación de la edad); la ubicación geográfica de la localización (intersección de calles); si se trataba de individuos aislados o grupos; la tenencia de animales; las características del lugar elegido para pernoctar (si duermen bajo puentes, terrenos baldíos, plazas, etc.); la posesión de pertenencias (mantas o trapos, nylon o maderas, alimentos, etc), entre otras. Asimismo, cada censista tenía la posibilidad de registrar información adicional. A diferencia del instrumento utilizado por el Gobierno de la Ciudad, este contempló preguntas cualitativas sobre información sociodemográfica general, la trayectoria en calle, redes familiares o de apoyo, trabajo e ingresos, salud en general y de consumo, educación, acceso a derechos y participación y expectativas de superación de la situación de calle. Se establecieron los siguientes criterios de validez y confiabilidad, a los fines de evitar duplicaciones, en el caso de las encuestas completas, y doble registro de observados, en el caso de no haber aceptado responder, a saber: a) cotejó nombres y fechas de nacimiento de las personas encuestadas, y b) los encuestadores fueron parte de organizaciones que conocían el territorio asignado, así como a la mayoría de las personas en calle que allí se encontraban. ${ }^{+}$

Se realizaron dos sesiones de retroalimentación presenciales con la mesa de organizaciones participantes, en las cuales se discutió el contenido de las preguntas, así como su formulación. De este modo, se garantizó la apropiación del instrumento de recolección de datos y la participación de las organizaciones en todas las etapas del proceso de trabajo. Asimismo, se recibieron aportes por correo electrónico.

Para la aplicación del instrumento se trabajó en equipos de entre dos y tres personas, de manera que mientras una conversaba con el/la entrevistado/a, otra registraba en la planilla. La misma no estuvo diseñada para preguntar obligatoriamente en un orden secuencial, a modo de interrogatorio, sino que se iba recuperando la información de la conversación, según las estrategias de quienes censaran. Se prestó especial atención al modo de acercamiento con los/as posibles encuestados/as, de manera que fuera un facilitador más que un obstáculo para la realización del cuestionario. Ir a buscar a las personas a "sus espacios", "a sus lugares" es como abrir "una puerta imaginaria", y lo que se diga y cómo se diga podía hacer que "esa puerta" se cerrara o se abriera. Quienes participaron como encuestadores/ras refirieron que, al momento de la participación, fueron bien recibidos/as y que incluso "la gente los estaba esperando". Al finalizar la entrevista, se entregó un material con información básica sobre derechos, así como puntos de contacto con organizaciones participantes.

\section{Delimitación del recorrido y la importancia del enfoque territorial}

El relevamiento se realizó entre el 8 y 15 de mayo de 2017 en la Ciudad Autónoma de Buenos Aires mediante una metodología de barrido territorial, con un muestreo estratificado regionalmente. Se utilizaron los límites y la ubicación geográfica de la Ciudad Autónoma de Buenos Aires (http://www.buenosaires.gob.ar/laciudad/barrios) para organizar 48 circuitos, uno por cada uno de los barrios. Se trató de un estudio de amplio alcance ya que la estrategia consistió en el barrido de la totalidad de la Ciudad de Buenos Aires a través de la organización de un trabajo de campo que tuvo como objetivo garantizar la cobertura de la mayor cantidad de PSC posible.

Cada barrio o circuito se dividió en un conjunto de manzanas, el cual fue recorrido por un equipo de censistas según los siguientes criterios:

\footnotetext{
4 Reconociendo que no hay pruebas perfectas, sin error o sin repetición, siguiendo a Bronfenbrenner (1997) se utilizó como criterio lo que se conoce como "validez ecológica": una investigación se considera como válida ecológicamente si se lleva a cabo en un ambiente naturalístico y con objetos y actividades de la vida de cada día. El 80\% de los/as encuestadores/as conocía el barrio, según refieren en el cuestionario de evaluación construido ad hoc.
} 
a) Los límites de la zona a recorrer (detalle de calles y avenidas) se determinaron a partir de la delimitación geográfica que establece el Gobierno de la Ciudad.

b) Cada zona asignada fue recorrida una vez en horario de la mañana, una por la tarde y una por la noche. Asimismo, un día de semana y otro de fin de semana. Esto responde a la distribución temporal del uso del espacio público por las personas en situación de calle, en función de cómo organizan la subsistencia.

c) El recorrido incluyó vía pública, bajo autopistas, espacios verdes, terminales de ómnibus, subtes y trenes, entradas de bancos, cajeros automáticos y hospitales, según se encontraran en la zona asignada. Se prestó especial atención a autos abandonados, containers y otros lugares posibles de pernocte no preparados para tal fin.

d) En la semana anterior al relevamiento, se realizó un recorrida por la zona asignada para identificar ranchadas $^{5}$ o personas asentadas en algunos lugares de la Ciudad, e informar y sensibilizar a las personas en situación de calle sobre el sentido del relevamiento y la importancia de sumarse de manera voluntaria a responder. Se entregó un material de sensibilización construido a tal fin.

e) En el caso de visualizar a personas en situación de calle en la zona asignada que estuvieran durmiendo o que no quisieran participar del relevamiento en ese momento, se volvió a pasar una vez más. De lo contrario, se registró solo en la planilla de observación

f) En caso de presencia de niños, niñas y adolescentes sin la presencia de un adulto referente, no se aplicó el cuestionario. Sólo se registró la planilla de observación.

Los circuiros o barrios se organizaron en 4 zonas, cada una de las cuales tenía un coordinador de referencia. A su vez, cada circuito o barrio tuvo un referente responsable de acompañar en el recorrido y comunicar a los/as coordinadores/as de zona cualquier dificultad en el desarrollo del relevamiento o necesidad que tuviera el equipo (materiales, refrigerios, falta de cobertura, necesidad de voluntarios, etc.) Al ser la población en situación de calle un grupo dinámico y oculto, tal como se explicitó previamente, el modo de garantizar "encontrarla" no se limitó a una mayor cantidad de días de relevamiento ni mayor cantidad de recorridas por la misma zona, sino a la dimensión territorial del relevamiento. Los/as referentes de circuito fueron asignados teniendo en cuenta el trabajo territorial en el barrio y la experiencia en el trabajo con la problemática para intervenir como facilitador. Asimismo, también se sumaron personas en situación de calle como censistas o en otras acciones para garantizar el relevamiento.

\section{Entrenamiento de censistas}

Participaron 431 colaboradores-censistas que recorrieron las calles de la Ciudad, organizados en 48 equipos. En su mayoría fueron mujeres, pero también participaron personas trans y varones. Los equipos se conformaron de manera mixta: participantes de las organizaciones, vecinos que se incluyeron a través de la convocatoria por redes sociales y personas en situación de calle. Se sumaron estudiantes de carreras de trabajo social, comunicación y psicología. También participaron trabajadores del Ministerio Público de la Defensa, de la Defensoría del Pueblo y de la Auditoría General. En estos casos, también fue de manera voluntaria.

El proceso de capacitación incluyó:

- Reuniones semanales entre abril y mayo con los referentes de circuito

- Capacitación general para los colaboradores-censistas en la que participaron más de 300 personas.

- 22 réplicas de la capacitación general para nuevos colaboradores.

Es importante volver a mencionar que los recursos humanos y materiales para realizar los entrenamientos estuvieron cubiertos con los recursos propios y disponibles de las organizaciones. Asimismo, en el caso de los entrenamientos, quienes estaban más familiarizados con el instrumento y con

\footnotetext{
s Ranchada es un término local utilizado por las personas en situación de calle para referirse a un grupo de personas, que puede ser mixto o sólo de hombres, que se asientan en un mismo lugar durante cierto período de tiempo. Se observan colchones, elementos de cocina y de trabajo, así como otros que dan cuenta que las personas regresan a ese lugar. Por lo general no superan las 6 o 7 personas, puede haber niños y adolescentes, se establecen entre sus miembros dinámicas que reproducen una lógica familiar.
} 
estas metodologías, producto de sus formaciones previas, circularon por las distintas organizaciones. Este aspecto vuelve a imprimirle un carácter participativo y autogestivo al censo, que además se tradujo en saldos organizativos para quienes participaron (nuevos integrantes, socialización de saberes, fortalecimiento de redes)

\section{Construcción del informe}

Los datos fueron sistematizados a partir del soporte técnico del Paquete estadístico IBM-SPSS. El primer ordenamiento y carga de datos al sistema informático lo realizó un equipo técnico del Ministerio Público de la Defensa, tal como se explicitó previamente. Con la intención de preservar la intimidad de las personas que participaron y la confidencialidad, todas las planillas completas se encuentran en dicho organismo. Una vez consolidada la base de datos, su análisis e interpretación estuvo a cargo de un equipo de trabajo integrado por miembros de las organizaciones que participaron del relevamiento. El mismo fue seleccionado por el conjunto de las organizaciones, reconociendo la importancia de los enfoques situados para el análisis y la interpretación de los datos. Con el objetivo de promover un debate sobre los resultados, garantizar "el derecho a la voz" de los protagonistas y lograr una interpretación consensuada, los resultados se discutieron entre las organizaciones participantes previo a su presentación final. El informe preliminar, titulado "AMiNoMeContaron: Visibilizar para Humanizar" fue presentado en una conferencia de prensa el 19 de Julio de 2017, en el Ministerio Público de la Defensa, y contó con la presencia del Defensor General de la Ciudad y se encuentra disponible on line.

La diferencia de resultados fue significativa:

\begin{tabular}{|c|c|c|}
\hline & Relevamiento oficial & Censo Popular \\
\hline PSC en el espacio público & 1092 & 4394 \\
\hline PSC en red de alojamiento & & 1478 \\
\hline & 1092 & 5872 \\
\hline
\end{tabular}

Fuente de elaboracion propia

Un 52\% de las 4590 personas contactadas $(n=2366)$ durante la realización del relevamiento contestó la encuesta de manera completa (Informe Preliminar Censo Popular, 2017), es decir, de la cual hay información cualitativa.

El 86,5\% de las 4590 personas contactadas fue mayor de 18 años, de los cuales un $20 \%$ de las personas entrevistadas $(n=402)$ no tiene documento de identidad ni está realizando el trámite para obtenerlo. Del total de población adulta contactada $(n=3981)$, un $49 \%$ estaba sola y un $12 \%$ se definió como grupo familiar. Asimismo, un 21,50\% $(n=856)$ fueron contactados en ranchadas.

De la población contactada que respondió el cuestionario $(\mathrm{N}=2366)$, un $24,5 \%$ son mujeres, un $74,5 \%$ son varones y un $1 \%$ personas trans. Esto podría comprenderse en clave de los procesos de socialización de los géneros. Mientras que para las mujeres se registran algunas estrategias de supervivencia que retrasan la llegada a la calle (trabajo en casas de familia, ayuda por parte de familiares y amigos por ser mujeres, y más si tienen hijos) para los varones el estereotipo de proveedor podría impactar negativamente en la recepción y aceptación de ayuda. En el caso del colectivo trans es dable mencionar las dificultades para poder contactar a esta población en situación de calle, no sólo por ser de las más vulneradas en todos los aspectos y de las más olvidadas para las políticas públicas, sino por el permanente movimiento y cambio de lugar de asentamiento a los que se ven obligadas, debido a la multiplicidad de violencias que padecen (violencia policial, de vecinas/os, otras). Además, la gran mayoría de las mujeres trans se ven obligadas a

\footnotetext{
${ }^{6}$ Incluyen 594 niños y niñas, y se restaron personas que contestaron el cuestionario pero que se encontraban durmiendo en algún dispositivo transitorio. Por eso el $\mathrm{N}$ en el caso de los resultados cualitativos es mayor.
} 
estar en situación de prostitución, por no poder acceder a un trabajo formal, situación que hace que no se las pueda ubicar/contactar en un lugar fijo. Una ranchada o grupo de hombres puede llegar a estar asentada por mucho tiempo en un mismo lugar; en cambio esta situación no se da en la población trans ya que diferentes situaciones de violencia, hostigamiento y persecución se lo impiden.

\section{Discusión: Transformar la queja en participación}

Tal como se muestra con la realización del relevamiento popular sobre personas en situación de calle, esa dinámica de encuentros-desencuentros en el espacio público se convierten en una oportunidad analítica. Como sostienen Boy \& Perelman (2008), el encuentro de las diferencias (re)produce desigualdades sociales y pueden convertirse en conflictos urbanos. Las narrativas morales promovidas por los grupos intervinientes expresan las (des)igualdades y en sus contenidos se encuentran qué proyectos de ciudad, cómo debe ser el espacio público, cuáles son los usos (i)legítimos, quiénes pueden utilizarlo y quién merece vivir en la ciudad.

Reflexionar sobre este relevamiento, a partir de los aportes de la Psicología Social Comunitaria, permite definirlo como una intervención comunitaria. Recuperando el concepto de participación social en su dimensión valorativa, técnica o instrumental y ético-política, lo que se pone en evidencia es la tensión entre la producción de subjetividades controladas o subjetividades en resistencia.

Colocadas las PSC en la estructura social como quienes "deben ser cuidados y asistidos", tal como se expresa en estudios que dan cuenta de las representaciones sociales sobre las PSC por la red de asistencia (Di Iorio et al 2016), se configura un vínculo asimétrico constitutivo de las intervenciones psicosociales y comunitarias. Los posicionan como subjetividades que "piden auxilio", desconfiadas a priori, portadoras de una inmensa voluntad de ser aceptados. Sin desconocer las secuelas y el impacto que esas vivencias de expulsión y de estigmatización generan, esas subjetividades también resisten y se hacen visibles como cuerpos que desafían ser negados (no reconocidos) y negativizados (percibidos como un otro peligroso y amenazante). Por el contrario, incluir a las PSC como un actor social, supuso reconocerlas como sujetos de derechos, como poseedoras de un saber sobre sus padecimientos y que ponen en funcionamiento prácticas para sí mismos y con sus grupos de pares, fundadas en la solidaridad, el respeto y la dignidad, tal como se evidenció en el proceso del Censo Popular. Esto significa no obturar los propios marcos de referencia y dar lugar a que inventen su praxis de manera que produzcan aperturas en el sistema de subjetividades dominante. Todos los devenires singulares, todas las maneras de existir de modo auténtico [muchas de las cuales por lo general entran en conflicto con nuestras propias maneras de existir desde los segmentos de clase, étnica, género] chocan contra el muro de la subjetividad controlada. Gestionar los fenómenos de singularidad presentes en las situaciones en las que intervenimos implica hacer visibles esos cuerpos que desafían no ser reconocidos y resisten a ser percibidos como un otro peligroso y amenazante. Recorridos de resistencias constituyen un intento de resignificar la vida en esos territorios y de recuperar la memoria social y personal (Rolnik \& Guattari, 2013)

La relación encuentro-desencuentro con las PSC que significó el censo popular supuso un ir y venir, una interpelación. Eso no se da de modo automático, sino que exige un intercambio de posibilidades. Esos encuentros habilitan desplazamientos: hay impases y aperturas, hay rupturas y clausuras. Los encuentros son movimiento uno-nosotros-otros. Es entenderse con alguien sobre algo, es decir y dejarse decir, es una actividad reversible de apelaciones y de respuestas, que transforma a todos los interlocutores. Desde la perspectiva intersubjetiva, ese encuentro es simultáneamente con los otros, con el mundo, con uno mismo. Las consideraciones metodológicas, así como los aspectos ético-políticos que se explicitaron, permiten comprender las diferencias entre los datos oficiales y los modos en que las organizaciones y las propias personas en situación de calle definen su vida cotidiana. Pero también, permiten identificar que las intervenciones pueden generar opresión o emancipación, no solo en función de sus resultados sino del proceso en sí mismo. En este sentido, la dimensión política adquiere central relevancia, ya que el poder no es algo que afecta de tal o cual manera a las poblaciones en condiciones de vulnerabilidad con las que intervenimos, sino que el poder es constitutivo de nuestras prácticas de intervención, y se introduce en la forma en que pensamos acerca de con quienes trabajamos y en la forma en la que los tratamos (Prilleltensky \& Nelson, 2002) 


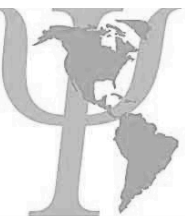

Lo vincular se configura como eje central de las intervenciones comunitarias, es el instrumento clave y el objetivo de una pedagogía del encuentro-desencuentro en una dialéctica de proximidad-

distanciamiento. En ese sentido, frente a un entramado asistencialista, debemos construir otros posicionamientos para lograr transformaciones que mejoren la calidad de vida de las comunidades y su acceso a bienes y derechos, de los que son continuamente expulsados. El desafío lo constituye reflexionar sobre nuestras prácticas e indagar sobre sus efectos, incorporando la problematización sobre las relaciones de poder. Problematizar los límites de las intervenciones psicosociales hegemónicas, se traduce en la posibilidad de construir territorios de intervención que promuevan transformaciones subjetivas y colectivas, que se traduzcan en la ampliación de derechos. Tal como sostiene Martín-Baró (1990), "el problema de fondo no está en los individuos, sino en las relaciones traumatógenas propias de un sistema (...) [Por lo tanto, las intervenciones] deben dirigirse también, y muy primordialmente, a la relación, a esos vínculos grupales que constituyen la normal anormalidad" (p. 246). 


\section{Referencias}

AMiNoMeContaron. Informe Preliminar Censo Popular (2017) Disponible en: https://proyecto7.org/acciones/censo-popular-de-personas-en-situacion-de-calle/

Bastos, F.I. \& Bertoni, N. (2014) Pesquisa Nacional sobre o uso de crack. Quem são os usuários de crack elou similares do Brasil? Quantos são nas capitais brasileiras? Rio de Janeiro: ICICT/FIOCRUZ

Boy, M. \& Perelman, M. (2008) Los Sin Techo de Buenos Aires. Ciudades. Las múltiples manifestaciones de la pobreza, 78, 2-7.

Boy, M. (2012) Políticas públicas que atienden a los adultos que viven en las calles de Buenos Aires: de la inserción social a la represión, 1997-2011. Intersticios: Revista Sociológica de Pensamiento Crítico, 6 (2). Disponible en http://www.intersticios.es/article/view/10110

Bustelo, E. \& Minujin, A. (1997) La política social esquiva. En Larin, R.; Kruijt, D; Tijseen, L. (eds.) Pobreza, exclusión y política social. (pp. 237-261) Costa Rica: Flacso.

Di Iorio, J., Rigueiral, G. et. al (2014) En busca de un lugar: espacio social vivido, construcción de realidad $y$ de identidad en personas en situación de calle. VI Congreso Internacional de Investigación y Práctica Profesional en Psicología XXI Jornadas de Investigación Décimo Encuentro de Investigadores en Psicología del MERCOSUR. Facultad de Psicología - Universidad de Buenos Aires, Buenos Aires. Disponible en: https://www.aacademica.org/000-035/496.pdf

Di Iorio, J. (2016) Perspectiva psicosocial de las vulneraciones sociales: diferencias económicas, desigualdades jurídicas, desafiliaciones sociales. En Wainsten, M. (comp.) Escritos de Psicología Social. (pp.175-188) Buenos Aires: JCE

Di Iorio, J.; Seidmann, S. et. al (2017) Construyendo comunidad: investigación-acción con personas en situación de calle en la Ciudad de Buenos Aires. PIUBAMAS. Universidad de Buenos Aires. Disponible

en http://cyt.rec.uba.ar/sitios/piubamas/SiteAssets/Documentos\%20del\%20Sitio/CONTRIBUCIONES/ Contribuciones\%202017_Di\%20Iorio\%20et\%20al.pdf

Dirección General de Estadística y Censos del Gobierno de la Ciudad de Buenos Aires. Disponible en https://www.estadisticaciudad.gob.ar/eyc/?cat=166

Donda Perez, V. y otros Contra GCBA sobre Amparo. Juzgado en lo contencioso administrativo $\mathrm{N}^{\mathrm{o}} 4$, Poder Judicial, Ciudad de Buenos Aires. Disponible en http://www.saij.gob.ar/gobierno-ciudadbuenos-aires-debera-realizar-relevamiento-personas-situacion-calle-nv15216-2016-0810/123456789-0abc-612-51ti-lpssedadevon

Goffman, E. (2003) Estigma. La identidad deteriorada. Buenos Aires: Amorrortú (Ed. original 1963)

Guattari, F. \& Rolnik, S (2013) Micropolítica. Cartografías del deseo. Buenos Aires: Tinta y Limón.

Instituto Nacional de Estadística y Censos. (2015). Censos, Encuestas y Registros Administrativos

Kessler, G. \& Merklen, D. (2013) Una introducción cruzando el Atlántico. En R. Castel, G. Kessler, D. Merklen \& N. Murard Individuación, precariedad, inseguridad. ¿Desinstitucionalización del presente? (pp. 9-31) Buenos Aires: Paidós.

Lambert, E. \& Wibel, W., (1990). Introduction. En E. Y. Lambert (Ed.) The collection and interpretation of date from hidden populations. (pp.15-27) National Institute on Drug Abuse Research Monograph Series 98. DHSS publication number (ADM) 90-1678, Washington, DC

Ley N $\mathrm{N}^{\circ}$ 3706/11 de Protección de derechos de las personas en situación de calle, Ciudad de Buenos Aires. Argentina

Martín-Baró, I. (1998) (comp.) Psicología social de la guerra: trauma y terapia. El Salvador: UCA Editores.

Molina, C. E. (2013). ¿Qué es eso de la interseccionalidad? Aproximación al tratamiento de la diversidad desde la perspectiva de género en España. Investig Fem, 3, 203-222.

Ochoa, C. (2015). Muestreo no probabilístico: Muestreo por conveniencia. Mexico: http://www. netquest. com/blog/es/muestreopor-conveniencia 


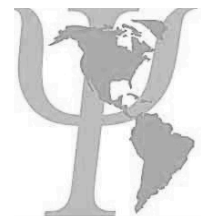

Palleres, G. (2012) Derecho a la ciudad: personas sin hogar en la ciudad de Buenos Aires. Dimensiones del hábitat popular latinoamericano. En Bolivar \& Espinosa (coord.) Dimensión del habitat popular Latinoamericano. Quito: FLACSO Ecuador. Disponible en http://bvsde.org.ni/clacso/publicaciones/gthi2.pdf

Pobreza y desigualdad por ingresos en la Argentina urbana 2011-2016. Observatorio de la Deuda Social Argentina, Pontificia Universidad Católica Argentina. Disponible en http://www.uca.edu.ar/uca/common/grupo68/files/2017-Observatorio-Informe-Pobreza-DesigualdadPor-Ingresos-2010-2016.pdf

Prilleltensky, I.\& Nelson, G. (2002) Doing Psychology Critically. Making a Difference in Diverse Settings. New York: Palgrave Macmillam

Rosa, P. (2012) Pobreza urbana y desigualdad: La asistencia habitacional a las personas en situación de calle en la Ciudad de Buenos Aires.En Bolívar, T. y Erazo Espinoza, J., Hábitat Popular e Inclusión Social. Quito: CLACSO.

Sawaia, B. (2011) As artimanhas da exclusão. Analise psicossocial e ética da desigualdade social. Vozes: Petrópolis, RJ

Seidmann, S.; Azzollin, S.; Thomé. S. \& Di Iorio, J. (2009) Prácticas y saberes de la vida cotidiana: las representaciones sociales de quienes viven en situación de calle. IV Congreso Marplatense de Psicología. Universidad Nacional de Mar del Plata, Mar del Plata.

Wacquant, L. (2010) Las dos caras de un gueto. Ensayos sobre marginalización y penalización. Buenos Aires: Siglo XXI Editores. 